\title{
Unified performance measures in network localization
}

\author{
Thorsten Nowak ${ }^{*}$ (D), Markus Hartmann and Jörn Thielecke
}

\begin{abstract}
With the evolving Internet of Things, location-based services have recently become very popular. For modern wireless sensor networks (WSNs), ubiquitous positioning is elementary. Hence, the demand of everlasting and low-cost sensor nodes is rapidly increasing. In terms of energy-efficiency, received signal strength (RSS)-based direction finding is a prospective approach providing location information in low-power sensor networks. Unfortunately, RSS-based direction finding is, as radio-based localization is in general, prone to multipath propagation of the wireless channel. Therefore, the impact of multipath fading as well as all other error source have to be modeled correctly and have to be considered in the design of a locating WSN.

In this paper, we derive the classical Cramér-Rao Lower Bound (CRLB) for RSS-based direction-of-arrival (DOA). The drawbacks of the classical CRLB and its influence on the optimal network topology are discussed. The CRLB indicates that the minimum variance unbiased estimator (MVUE) does not exist for the problem of RSS-based DOA due to the nature of its measurement function. Hence, beyond the CRLB, we derive performance metrics for the maximum likelihood estimator (MLE) and compare position estimation errors for the MVUE and the MLE for different network topologies. Since both approaches, the CRLB and the maximum likelihood (ML) limits, are not capable of handling ambiguities, we introduce another measure for the variance of a measurement and its corresponding position estimate based on information theory. This way, the amount of information for a set of RSS measurements can be quantified exactly, even in the case of ambiguous probability densities. Thus, the proposed technique gives a holistic view on the information obtained from sensor measurements which can be utilized for network topology optimization.
\end{abstract}

Keywords: Information theory, Estimation theory, Cramér-Rao lower bound (CRLB), Localization, Wireless sensor network (WSN), Network localization, Location sensors

\section{Introduction}

The use of wireless sensor networks (WSNs) is rapidly increasing these days. Also, new aspects of WSNs, like energy-efficiency and location-awareness, are gaining more and more attention. There is a vast number of applications for location-aware WSNs, e.g., smart metering [1], collision avoidance [2], or animal tracking [3], just to name a few of them. In all of these examples, the sensor information is almost meaningless without any position information. Hence, localization is a core feature of today's sensor networks [4].

Radio-based localization is one of the most popular techniques to provide location information in [5]. There is one thing all radio-based localization systems have in

*Correspondence: thorsten.nowak@fau.de

Institute of Information Technology, University of Erlangen-Nürnberg, Erlangen, Germany common: the wireless radio channel. Precise localization would be an easy challenge without its impairments of the wireless propagation channel. To design locating WSNs in an optimal way, these impairments have to be modeled in order to analyze the performance of the network localization. A straightforward approach is to model the errors of each sensor and combine the error distributions of all sensors with regard to the geometry of the WSN [6]. Estimation theory allows to compute the expected variance for the sensors evaluating the Cramér-Rao Lower Bound (CRLB). In a similar manner, the position CRLB can be computed for noisy sensor estimates, e.g., ranging and direction finding, for a location estimate within a sensor network [7].

The effect of multipath and non-line-of-sight propagation on the precision of the position estimates has been extensively analyzed in [8]. Shen et al. consider the use of 
prior knowledge of the user's position [8]. Prior knowledge may be integrated with the information-theoretic performance metric presented in this paper. As shown by [8], the use of prior information is reasonable, as it may be provided by e.g. maps. Hence, performance metrics for WSNs should be capable of incorporating prior information. In [9], the impairments due to multipath propagation are characterized. Multipath fading results from destructive and constructive interference of different propagation paths at the receiver. When ranging is considered with narrowband systems, multipath components can not be resolved. This inherently leads to biased estimates. The same is true for the spatial domain in received signal strength (RSS)-based direction finding, where multipath propagation affects the accuracy of direction-of-arrival (DOA) estimates. In [10], anchored and anchor-free localization is compared. According to [10], accurate position estimates require for a good local geometry. Furthermore, in their conclusion, the authors state that a tool for the identification of the bottleneck in localization would be useful. The information-theoretic approach, presented in this paper, provides exactly that type of information, i.e., quantifying the information gain of additional sensor nodes. In many fields, there has been a revival of information theory. Information-theoretic measures are used to optimize MIMO radar waveforms [11]. The loss of sub-Nyquist sampling has been characterized applying information theory $[12,13]$. Within the context compressive sensing, fundamental limits [14] and bounds for kernel-based time delay estimation [15] have been derived based on information theory.

Although there are many manifestations of radio localization systems, the technique addressed in this paper is based on RSS measurements, more specifically RSS-based direction finding [16]. Although we focus on RSS-based
DOA estimation, the presented framework is applicable in general. Hence, it may be applied to cooperative ranging or time-difference-of-arrival (TDOA)-based localization systems. RSS-based DOA is a promising approach for several reasons. Field strength measurements do not demand for complex signal processing. In contrast to time-of-flight measurements, RSS measurements do not require exhaustive synchronization of the sensors. RSS-based DOA is a range-free approach. Hence, it does not depend on prior knowledge of the emitted power of the transmitter or the path loss exponent of the wireless channel [17]. A representative of such a RSS-based localization system is the $B A T S^{1}$ system [18]. The BATS system is an energyefficient sensor network designed for wildlife monitoring, more precisely the tracking of bats. Within the WSN, position information is obtained from RSS measurements [19] that are gathered in the sensor network and stored on a central computing unit, cf. Fig. 1.

For optimal deployment of the wildlife monitoring network in the woods, a performance analysis of the network localization for a given topology is essential. Therefore, we provide a position CRLB for RSS-based DOA for the antenna array utilized in the BATS project [20]. Due to the nonlinearity, more specifically the missing curvature [21] of the measurement function for the problem of RSSbased direction finding, applying the classic CRLB results in an unbounded variance for some signal directions. This unbounded variance indicates the minimum variance unbiased estimator (MVUE) does not exist. Besides that, RSS-based DOA, as many other fundamental estimation problems, such as DOA estimation, frequency and phase estimation, involve parameters that are of a cyclic nature. There exist modifications of the CRLB that address estimation of periodic parameters [22, 23]. However, periodicity is still on the downside of the CRLB.

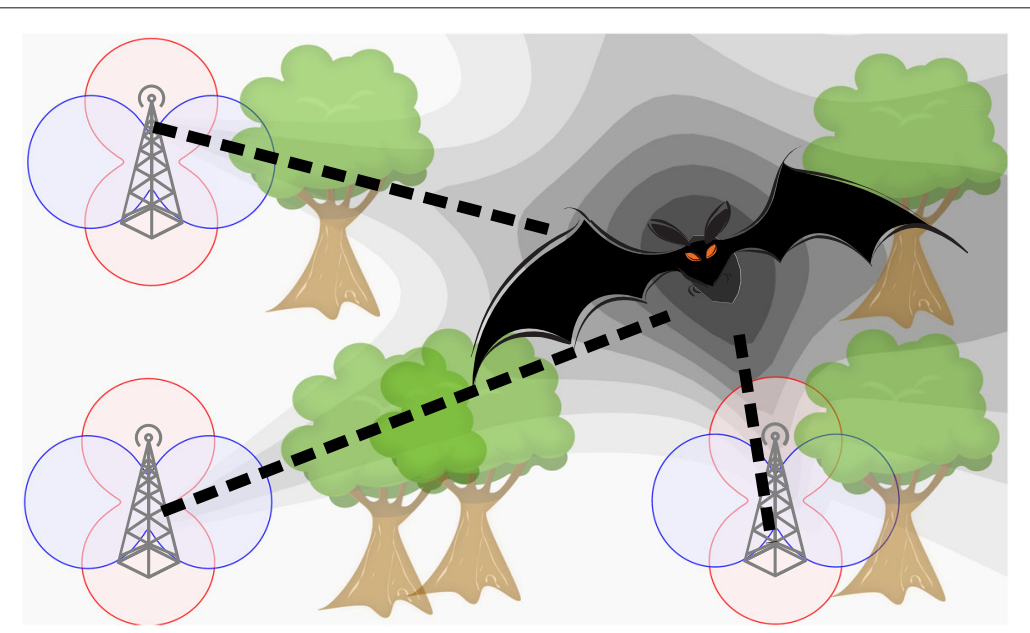

Fig. 1 Tracking bats in the wild: The bat's position, more precisely its location PDF, is inferred from RSS-based DOA measurements 
As a consequence, a performance metric based on the error distribution of the maximum likelihood estimator (MLE) is derived in [24]. The Bayesian estimation approach allows to integrate non-informative priors [21] in order to cope with cyclic parameters. This approach reveals more consistent results in terms of the position's mean squared error (MSE). In [20, 24], bounds for RSS-based DOA have been derived for an MVUE and a biased estimator, the MLE, showing completely different results. Results from estimation bounds significantly affect the optimization of network topology in local positioning [7]. As shown in [24], choosing another estimation bound entirely changes the constraints of the sensor arrangement.

However, the maximum likelihood (ML) approach still lacks the capability to handle ambiguities that inherently arise in RSS-based DOA. Thus, we introduce a novel approach to characterize the information content for multimodal measurement error distributions. Based on information theory, the entropy of sensor measurements is quantified. Finally, the entropy of a set of measurements retrieved by a WSN is related to a corresponding variance of unimodal measurement distribution. Information theoretic measures, i.e., entropy and mutual information, allow for performance assessment of locating WSNs in a holistic manner incorporating local precision and the impact of ambiguities. Utilizing the proposed informationtheoretic performance metric allows for network topology optimization in heterogeneous sensor networks with arbitrary measurement error probability density functions (PDFs).

The main contributions of this paper are summarized as follows.

- A review of RSS-based direction finding utilizing coupled dipole antennas is given.

- We present the classical CRLB for RSS-based DOA. Furthermore, the impact of network topology, i.e., the arrangement of the sensor nodes, on the position resolution is assessed for the MVUE by applying the 2-D position CRLB.

- Estimation errors for the MLE are derived for RSS-based direction finding. Again, the influence of the topology of the WSN is analyzed. The error bound for the MLE is more consistent compared to the MVUE and features a significantly smaller mean squared position error.

- Both findings above give indications of position resolution for the given networks. However, inherently arising ambiguities in RSS-based DOA are not considered. Therefore, we introduce informationtheoretic measures for the analysis of direction finding in WSNs. These information-theoretic measures allow for joint analysis of resolution and ambiguities. Hence, they provide a holistic view on the performance of the locating WSNs.

- We provide a design tool, based on mutual information, for the comparison and optimization of different network topologies considering different sensors types (e.g., unambiguous and ambiguous DOA sensors). The use of mutual information allows to exactly quantify the loss of information due to the presence of ambiguities, whereas the CRLB gives no useful information in that comparison.

The remainder of this paper is organized as follows. In Section 2, a brief review of RSS-based direction finding is given. Section 3 elaborates on the classic CRLB deriving the CRLB for RSS-based direction finding and corresponding position estimates with concluding remarks on network topology as well as its drawbacks. Performance limits of MLEs for RSS-based DOA are proposed in Section 3.2. These are compared to the result from classical CRLB analysis. In Section 4, a unified approach to quantify measurement uncertainty based on information theory is introduced and illustrated for multimodal measurement PDFs. A network design tool for optimal node arrangement is presented in Section 5. Furthermore, the impact of ambiguous sensors in different network localization scenarios is discussed. Section 6 concludes this paper.

\section{A primer on RSS-based direction finding}

For this paper, we consider RSS-based DOA estimation applying coupled dipole antennas [24]. Furthermore, it is assumed that localization takes place in the horizontal plane orthogonal to the two dipoles. Presuming a perfect linear dipole array, the radiation pattern of the dipoles in the horizontal plane (i.e., $\theta=90^{\circ}$ ) is constant over all impinging signal angles in azimuth $\phi \in[0,2 \pi]$. Hence, the radiation pattern for $N$ dipoles at a distance of $d=1 / 2 \lambda$ is given by the array factor [25]

$$
\mathrm{AF}=\sum_{i=0}^{N-1} c_{i} \cdot \exp \left(-j \cdot 2 \pi \sin (\theta) \cdot d_{i} \cos (\phi)\right),
$$

where $d_{i}$ are the corresponding distances of the dipole elements, $\lambda$ is the wavelength, and $c_{i}$ is the coupling factor. Considering only two dipoles at distances $d_{0}=0$ and $d_{1}=d$ and $\theta=90^{\circ}$ the array factor reduces to

$$
\mathrm{AF}=c_{0}+c_{1} \cdot \exp (-j \cdot 2 \pi \cdot d \cos (\phi)) .
$$

For the considered antenna array, the dipoles are coupled in phase. Hence, the radiation pattern is given by

$$
g(\phi)=1+\exp (-j \cdot 2 \pi \cdot d \cos (\phi)) .
$$

We define the radiation power patterns $G(\phi)$ (in $\mathrm{dB}$ ) by

$$
G(\phi)=10 \lg \left|g_{a}(\phi)\right|^{2}
$$


With two identical antennas rotated by $90^{\circ}$ towards each other, the gain difference function is expressed by

$$
\Delta G(\phi)=G(\phi)-G(\phi+\pi / 2) .
$$

The patterns described above are sketched in Fig. 2. Gain patterns for the rotation angles of $0^{\circ}$ and $90^{\circ}$, respectively, are depicted. DOA estimation yields minimum variance at a high gradient of the gain difference function, as shown in the next section.

The RSS at a receiver $a$ for a transmitted signal with power $P_{\mathrm{TX}}$ can be computed as follows

$$
P_{\mathrm{RX}, a}=P_{\mathrm{TX}}-L+G_{\mathrm{TX}}+G(\phi)+w_{a},
$$

with $L$ denoting the bulk path loss. $G_{\mathrm{TX}}$ and $G_{a}(\phi)$ are transmit and receive antenna gain, respectively. When considering a single signal source, i.e., no multipath propagation, the received signal strength difference is given by

$$
\Delta P_{\mathrm{RX}}=\Delta G(\phi)+w
$$

due to the fact that both channels are stimulated by the same transmit power and exhibit equal path loss. Thus, the gain difference function does not depend on transmit power and path loss. Hence, it maybe estimated without prior knowledge of the the path loss exponent and the power emitted by the transmitter. This fact is, in contrast to range-based localization based on RSS, a major benefit of RSS-based DOA estimation.

\section{Conventional methods for performance assessment}

Estimation theory is a core essential of many modern signal processing system. Those include, but are not limited to, radar, image analysis, communications, and localization. In estimation theory, a parameter $\theta$ is inferred from a set of measurement $\mathbf{z}$. In parameter estimation, two basic concepts have to be distinguished [21]:

Deterministic parameter estimation Parameters are assumed to be deterministic but unknown in classical estimation theory, whereas in Bayesian estimation theory, the parameter, that is to be estimated, is assumed an to be a random variable (RV). Thus, the data, in classical estimation, is described by a PDF of the form $p(\mathbf{z} \mid \theta)$.

Bayesian estimation In contrast to that, in Bayesian estimation theory, is described by joint PDF $p(\mathbf{x} ; \theta)=$ $p(\mathbf{z} \mid \theta) p(\theta)$ that is composed of the measurement likelihood $p(\mathbf{z} \mid \theta)$ and the prior PDF $p(\theta)$. Hence, Bayesian estimation theory allows to incorporate prior knowledge on the parameter $\theta$.

In the sequel, the CRLB for classic parameter estimation is discussed. For the Bayesian approach, we derive an ML error bound for power-based direction finding.

\subsection{Cramér-Rao Lower Bound}

In parameter estimation, not only the estimate itself is of valuable interest, but also the distribution of its errors. Hence, it is desirable to quantify the deviation of the estimate from the true parameter value. Basically, estimators should be unbiased on the one hand. On the other hand, they should provide a minimum variance. For the MVUE, the Cramér-Rao Lower Bound gives a lower bound on the variance of an estimator with a zero-mean error [26].

When recalling the alternative form of the CRLB, [21] its respective limit on the variance is defined by

$$
\operatorname{var}(\hat{\theta}) \geq E\left[\left(\frac{\partial \ln p(z \mid \theta))}{\partial \theta}\right)^{2}\right]^{-1}
$$
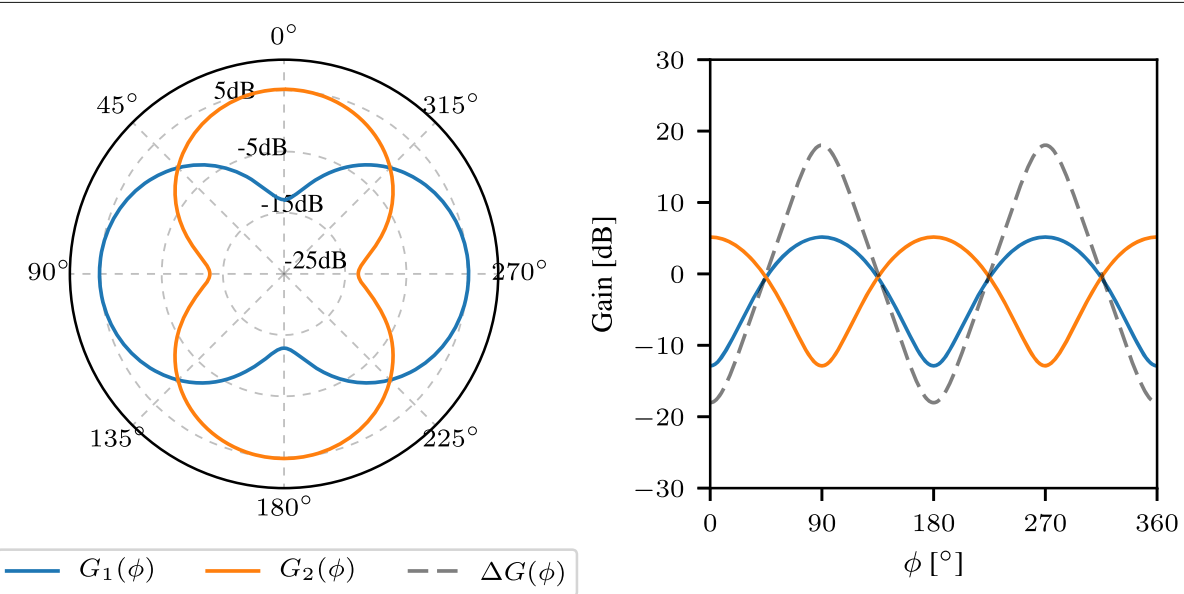

Fig. 2 The gain functions for both antenna configurations, rotated by $0^{\circ}$ and $90^{\circ}$, are depicted in blue and orange, respectively. Also, the gain difference function (dashed gray) and its derivative (dotted green) are depicted 
for a parameter estimate $\hat{\theta}$ observed by noisy measurements $r$. In case of an unknown parameter, $\theta$ of a deterministic signal observed in additive white Gaussian noise (AWGN) by a series of observations

$$
r[n]=f[n ; \theta]+w[n] \quad n=0,1, \ldots, N-1
$$

the general CRLB above can be strapped down to

$$
\operatorname{var}(\hat{\theta}) \geq \frac{\sigma_{z}^{2}}{\sum_{n=0}^{N-1}\left(\frac{\partial f[n ; \theta]}{\partial \theta}\right)^{2}},
$$

where $w \sim \mathrm{N}\left(0, \sigma^{2}\right)$. Equation 10 simplifies to

$$
\operatorname{var}(\hat{\theta}) \geq \frac{\sigma_{z}^{2}}{\left(\frac{\partial f(\theta)}{\partial \theta}\right)^{2}}
$$

for a single observation in presence of a AWGN.

\subsubsection{DOA Cramér-Rao Lower Bound}

The RSS measurements retrieved by the WSN are impaired by noise resulting from fading effects of the wireless propagation channel. Results from a channel measurement campaign [27] show that noise on RSS difference measurements is log-normal distributed with a constant variance over different ranges between transmitter and sensor node. When observing RSS difference measured in $\mathrm{dB}$, the CRLB estimating DOA by RSS difference measurements is expressed by [20]

$$
\operatorname{var}(\phi) \geq \frac{\sigma_{r}^{2}}{\left(\frac{\partial \Delta G(\phi)}{\partial \phi}\right)^{2}} .
$$

The resulting CRLB for RSS-based direction finding for the BATS sensor node is depicted in Fig. 3. It can be easily seen that the variance of the DOA estimate strongly depends on the direction of the impinging signal. Moreover, the variance is unbounded for signal directions near $\phi \approx k \frac{\pi}{2}$. This disadvantageous behavior can be explained by the gradient of the gain difference function $\Delta G(\phi)$ which approaches zero for the corresponding locations. Equivalently, it can be stated that the measurement function has no curvature [21] at the considered positions. Summarizing the results so far, the variance of unbiased DOA estimates inherently depends on the direction of the signal source. Hence, position estimation errors will also be dependent on the location of the tracked object.

\subsubsection{Position Cramér-Rao Lower Bound}

The CRLB for DOA estimates has been derived in the section above. With the results for the variance of the DOA sensors, the variance of the corresponding position estimate can be computed applying the position CRLB $[20,28,29]$ for a set of sensor nodes. In the scope of the paper, DOA estimates are considered in the horizontal plane. Hence, position state space is $2-\mathrm{D}$. However, the concepts presented here may be easily extended to 3-D position space. The covariance of a position estimate

$$
\operatorname{var}(\hat{\mathbf{x}})=E\left[(\hat{\mathbf{x}}-\mathbf{x})(\hat{\mathbf{x}}-\mathbf{x})^{\mathrm{T}}\right]
$$

is considered. As position estimation in general is vector parameter estimation, the Fisher information matrix (FIM) [21] can be stated as

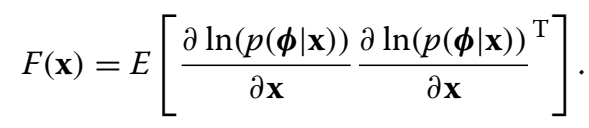

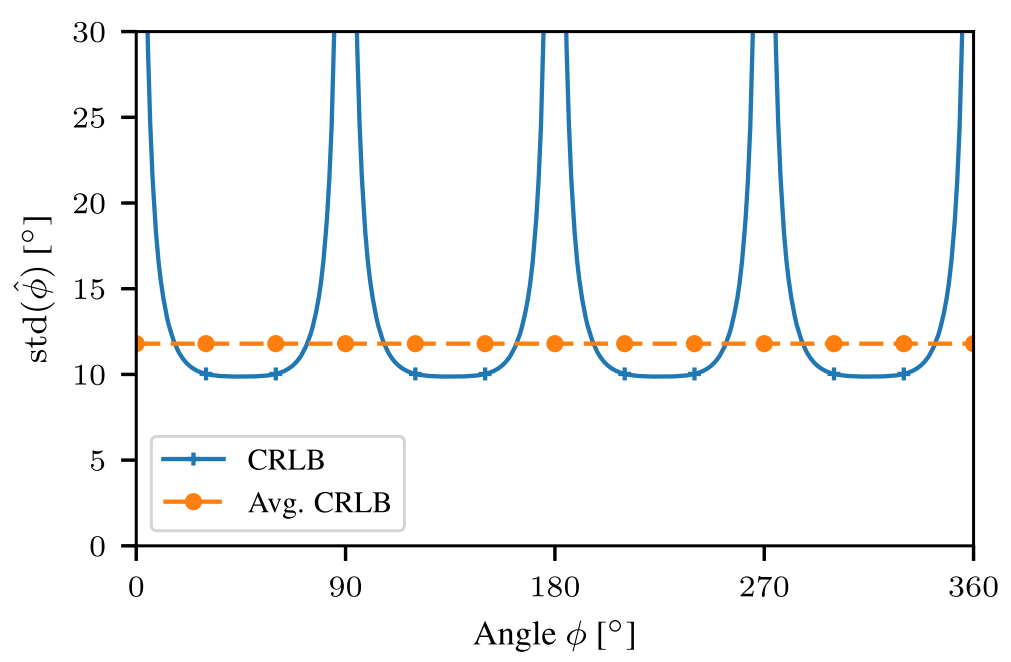

Fig. 3 CRLB for RSS-based DOA estimation. Apparently, DOA estimation variance depends on the direction of the received signal. For a received signal direction of $\phi \approx k \frac{\pi}{2}$, the variance approaches infinity. This is quite obvious, as the gain difference function $\Delta G(\phi)$ is close to zero in that range. Therefore, network topologies, especially node orientation, matters 
$\phi$ denotes a vector of DOA measurements and $p(\boldsymbol{\phi} \mid \mathbf{x})$ is the measurement likelihood for a given location $\mathbf{x}=$ $[x, y]^{\mathrm{T}}$ of the tracked object.

The joint likelihood for all sensors is given by the product of the likelihoods for the DOA observations $\phi_{k}$ at all nodes of the network. The log-likelihood is expressed by

$$
\ln (p(\boldsymbol{\phi} \mid \mathbf{x}))=\sum_{k} p\left(\phi_{k} \mid \mathbf{x}\right)
$$

where the subscript distinguishes the sensor nodes contributing to the estimate. For DOA measurements in presence of noise, the measured angles $\phi$ for a given position $\mathbf{x}$ are given by

$$
\phi=g(\mathbf{x})+w
$$

with the measurement function

$$
g_{k}(\mathbf{x})=\tan ^{-1} \frac{\Delta y_{k}}{\Delta x_{k}}
$$

where $\Delta x_{k}=x-x_{k}$ and $\Delta y_{k}=y-y_{k}$. The noise processes $\boldsymbol{w}$ are assumed to be mutually independent Gaussian random variables. The likelihood function for a single DOA measurement $p\left(\phi_{k} \mid \mathbf{x}\right)$ neglecting some scaling factors can be written as

$$
p\left(\phi_{k} \mid \mathbf{x}\right) \propto \exp \left(-\frac{1}{2 \sigma_{\mathrm{DOA}}^{2}(\mathbf{x})}\left[\phi_{k}-g_{k}(\mathbf{x})\right]^{2}\right) .
$$

It has to be noted that the variance $\sigma_{\mathrm{DOA}}^{2}(\mathbf{x})$ of the DOA estimates is dependent on the angle of the impinging signal. Thus, it is a function of the location of the transmitter. We are now able to compute the FIM merging Eqs. (14), (15), and (18) and resulting in

$$
F(\mathbf{x})=\left[\begin{array}{ccc}
\sum_{k} \frac{\Delta y_{k}^{2}}{\sigma_{\mathrm{DOA}}^{2}(\mathbf{x}) d_{k}^{4}} & -\sum_{k} \frac{\Delta x_{k} \cdot \Delta y_{k}}{\sigma_{\mathrm{DOA}}^{2}(\mathbf{x}) d_{k}^{4}} \\
-\sum_{k} \frac{\Delta x_{k} \cdot \Delta y_{k}^{4}}{\sigma_{\mathrm{DOA}}^{2}(\mathbf{x}) d_{k}^{4}} & \sum_{k} \frac{\Delta x_{k}^{2}}{\sigma_{\mathrm{DOA}}^{2}(\mathbf{x}) d_{k}^{4}}
\end{array}\right],
$$

with $d_{k}=\left\|\mathbf{x}-\mathbf{x}_{k}\right\|_{2}$ for position estimation from DOA obtained from RSS measurements. The position estimation error bound for DOA-based localization in a WSNs is retrieved by evaluating the trace of the inverted FIM:

$$
\sigma_{\mathrm{POS}}^{2} \geq \operatorname{tr}\left(\boldsymbol{F}(\mathbf{x})^{-1}\right) .
$$

With help of the derived CRLB for network localization utilizing RSS-based direction finding, different network topologies can be assessed with respect to their localization performance Fig. 4.

\subsubsection{Optimal sensor node arrangement}

The variance of direction estimates significantly changes with the angle of the impinging signal with respect to the orientation of the antenna array. Hence, not only the node positions influence the localization performance, but also the orientation of the sensor nodes is of importance. To elaborate the impact of node orientation on the location errors, two network configurations with identical node positions but different orientation of receive antennas have been defined. For the analysis, the node orientation is defined as the rotation of the receive antenna array in the horizontal plane with respect to the $x$-axis of the Cartesian coordinate system. The optimal rotation angles can be found by a parameter search. Optimal node orientation is found by minimization of the mean CRLB over the considered area of interest. The mean CRLB for the two examined areas is depicted for different orientation angles in Fig. 5. Orientation of the sensors is $0^{\circ}$ and $35^{\circ}$ for the tested networks configurations 1 and 2, respectively. In both cases, the nodes have a spacing of $50 \mathrm{~m}$. In total, four nodes are used being arranged in a quadratic shape. A noise variance of $\sigma_{\Delta \mathrm{RSS}}=5 \mathrm{~dB}$ is assumed for the cross fading of the two antennas of the sensor nodes.

The position CRLB for both networks is visualized in Fig. 4a, b. For the first network, the position error bound is very inhomogeneous. This network yields promising performance for the center of the area of interest. But on the other hand, the position resolution is poor in the outer areas. Having a look at network 2, the errors are distributed in a much more uniform way compared to network 1. Therefore, network 2 features good average results for the whole area of interest. Simulation results are shown in Table 1 for the two network configurations. Average position errors are computed for networks 1 and 2 considering two different regions $x, y \in[10,40]$ and $x, y \in[-10,60]$. These numbers are in line with visual representation of $2 \mathrm{D}$ error distribution presented in Fig. 4a, b.

In sum, DOA dependent noise variance causes the position errors to be position dependent. This, in consequence, makes the network localization sensible to node orientation. In addition to the fact that node orientation significantly affects position errors, also the area of interest has to be considered thoroughly for the assessment of localization performance and the design of locating sensor networks.

\begin{tabular}{|c|c|c|}
\hline \multirow[b]{2}{*}{ Area of interest } & Network 1 & Network 2 \\
\hline & \multicolumn{2}{|c|}{ Avg. position error } \\
\hline$x, y \in[+10,40]$ & 6.33 & 8.89 \\
\hline$x, y \in[-10,60]$ & 11.18 & 8.85 \\
\hline
\end{tabular}

\subsubsection{Results and discussion}

Estimation variance significantly depends on the direction of the signal source when considering the MVUE for DOA estimation. Reflecting the results from above,

Table 1 Simulation parameters and results 


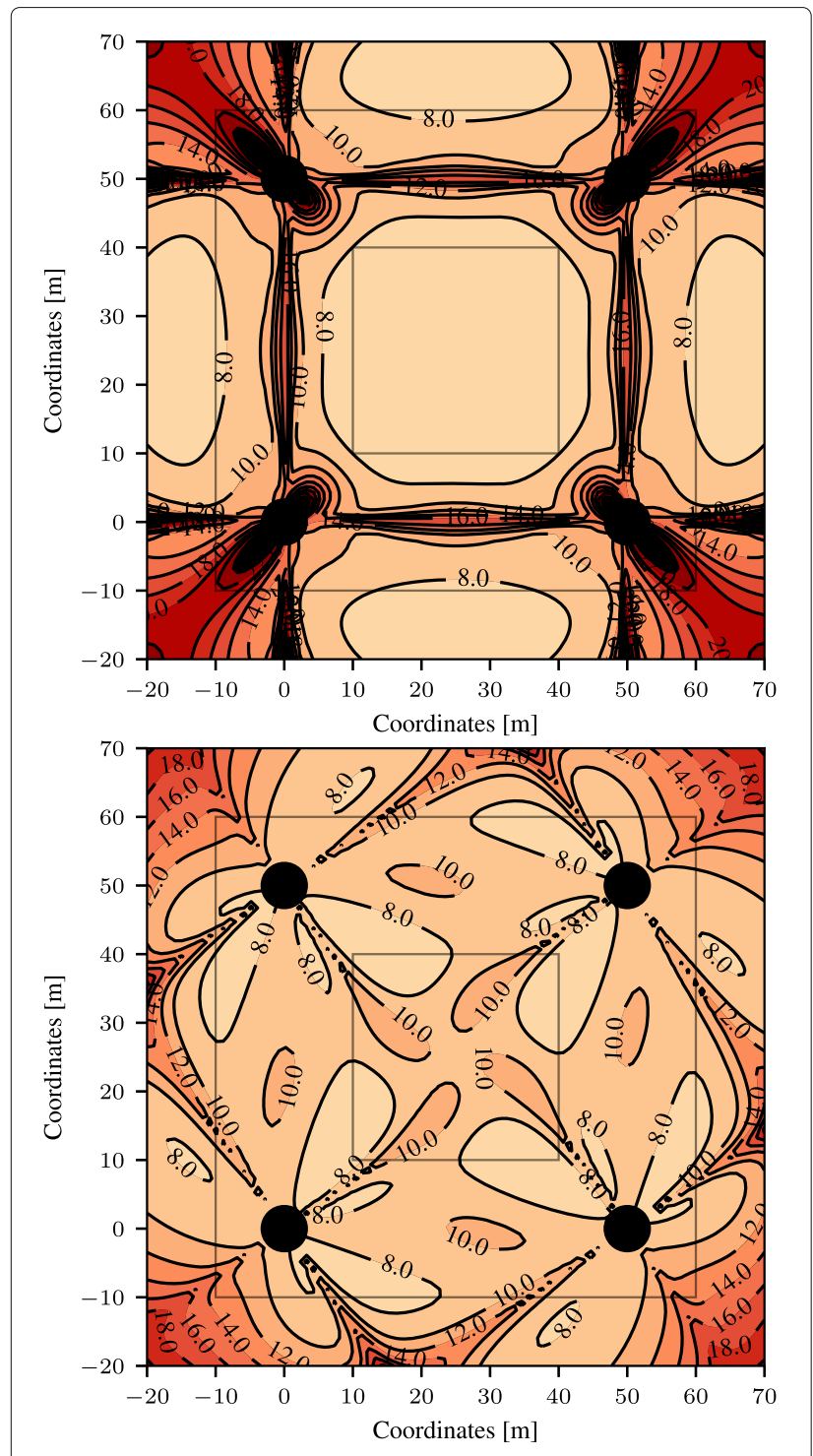

Fig. 4 CRLB for position estimation based on RSS-based DOA measurements depending on the node orientation. For both figures standard deviation is given in meters. a Position CRLB for a node orientation of $0^{\circ} \mathbf{b}$ Position CRLB for a node orientation of $35^{\circ}$

topology optimization of the WSN is not only subject to the antenna gain patterns and its orientation. It also significantly depends on the area of interest for the object that is to be tracked. Prior knowledge of the spatial probability density of the tracked object allows for an optimal design of the locating network which results in a decrease in position estimation errors.

However, due to missing curvature of the RSS-based DOA measurement function, the MVUE yields an unbounded variance for angle estimates at multiples of $90^{\circ}$. This indicates that the MVUE may not even exist. In the next section, we will have a look on the maximum likelihood estimator. Furthermore, the classic CRLB can not

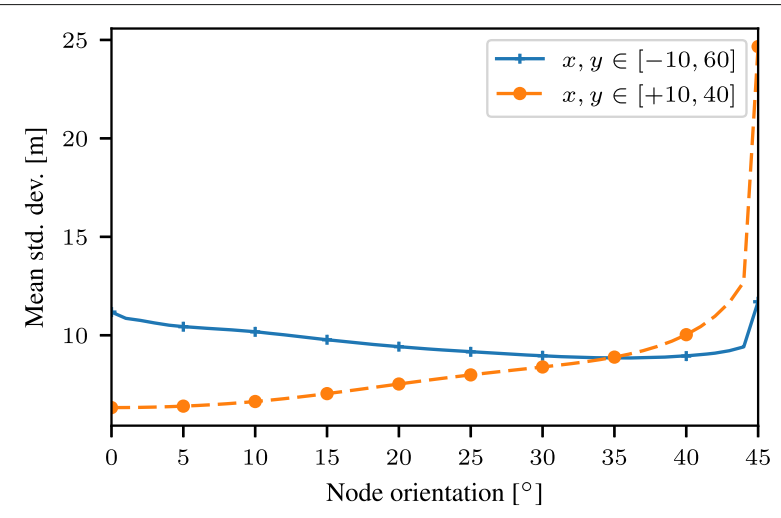

Fig. 5 Optimization of node orientation for the considered network. The lines denote the two considered areas of interest (cf. Fig. 4b)

handle ambiguities, which inherently exist in RSS-based DOA.

\subsection{Bayesian estimation error bound}

In general, the MLE $\hat{\theta}_{\mathrm{ML}}$ maximizes the likelihood function for a parameter of interest $\theta$ observed by noise measurements $r$ [30]

$$
\hat{\theta}_{\mathrm{ML}}=\underset{\theta}{\operatorname{argmax}} p(r \mid \theta) .
$$

We further assume that the parameter of interest is estimated for a particular realization of a random variable $\theta$. Taking the logarithm yields to

$$
0=\left.\frac{\partial}{\partial \theta} \ln (p(r \mid \theta))\right|_{\theta=\hat{\theta}_{\mathrm{ML}}} .
$$

For AWGN, we can write the following condition

$$
0=\left.(r-g(\theta)) \frac{\partial}{\partial \theta} g(\theta)\right|_{\theta=\hat{\theta}_{\mathrm{ML}}},
$$

where, in order to equate to zero, at least one of terms of the product has to be zero. As in general $\frac{\partial}{\partial \theta} g(\theta) \neq 0$, the inverse function $g^{-1}$ maximizes the likelihood function. Therefore, in the considered case, the MLE is simply given by the inverse of the measurement function.

$$
\hat{\theta}_{M L}(r)=g^{-1}(r)
$$

\subsubsection{Computation of posterior density}

In this section, the posterior density for a RSS-based angle estimate is computed. Again, we assume field strength difference measurements to be impaired by AWGN

$$
r=\Delta G(\phi)+w
$$

where $\phi$ is the azimuth angle of the impinging signal and $w$ is a AWGN process with $\mathcal{N}\left(0, \sigma_{r}^{2}\right)$. The likelihood function for the DOA estimation problem is given by [24] 


$$
P(r \mid \phi)=\frac{1}{\sqrt{2 \pi \sigma_{r}^{2}}} \exp \left[-\frac{1}{2}\left(\frac{r-\Delta G(\phi)}{\sigma_{r}}\right)^{2}\right] .
$$

Recalling (12) (cf. [20]), the CRLB for a DOA estimate from a single observation of two field strength measurements states as

$$
\operatorname{var}(\hat{\phi}) \geq \frac{\sigma_{r}^{2}}{\left(\frac{\partial(\Delta G(\phi))}{\partial(\phi)}\right)^{2}} .
$$

Noting again, the MVUE has an unbounded variance for $\phi$ approaching multiples of $90^{\circ}$ as shown as dashed line in Fig. 3. The variance to be unbounded makes sense due to the property of the gain difference function $\Delta G$. The function $\Delta G$ has no curvature [21] at multiples of $90^{\circ}$. From an applications point of view, it is not reasonable to make use of an estimator with unbounded variance. Moreover, the unboundedness of the variance for the MVUE indicates that the estimator does exist. Allowing the estimator to be biased allows for less variant estimates.

In order to assess the performance of the MLE, we compute the full posterior PDF that can be derived by applying Bayes' theorem:

$$
P(\phi \mid r)=\frac{P(r \mid \phi) P(\phi)}{P(r)} .
$$

Due to point and axial symmetry of the gain difference function, $\Delta G P(\phi)$ may be limited to $\phi \in\left[0,90^{\circ}\right]$ without loss of generality. As there is no prior knowledge on the distribution of the directions of a signal source, the prior density is given by a uniform distribution

$$
P(\phi) \sim \mathcal{U}(0, \pi / 2) \text {. }
$$

This PDF is a non-informative prior [21]. The marginal likelihood $P(r)$ for the RSS measurement results from

$$
P(r)=\int_{\phi} P(r \mid \phi) P(\phi) \partial \phi .
$$

For the antenna considered in the scope of this paper (cf. Fig. 2), the marginal density is shown in Fig. 6a. Referring to (28), the posterior is computed by normalizing the likelihood with the marginal density for the field strength measurements. Figure $6 \mathrm{~b}$ depicts the posterior PDF for RSS-based DOA. Exemplary posterior PDFs are sketched for some specific field strength measurements $r \in[0 \mathrm{~dB}, 12 \mathrm{~dB}, 18 \mathrm{~dB}, 24 \mathrm{~dB}]$.

\subsubsection{DOA estimation errors}

With the posterior density of an angle estimate, the MSE for RSS-based direction finding is derived in this section. Considering the MLE introduced in (24), the inverse function of the stated problem is

$$
\hat{\phi}=\Delta G(r)^{-1},
$$

where $\Delta G(r)^{-1}$ is the inverted gain difference function for the considered antenna array. However, there
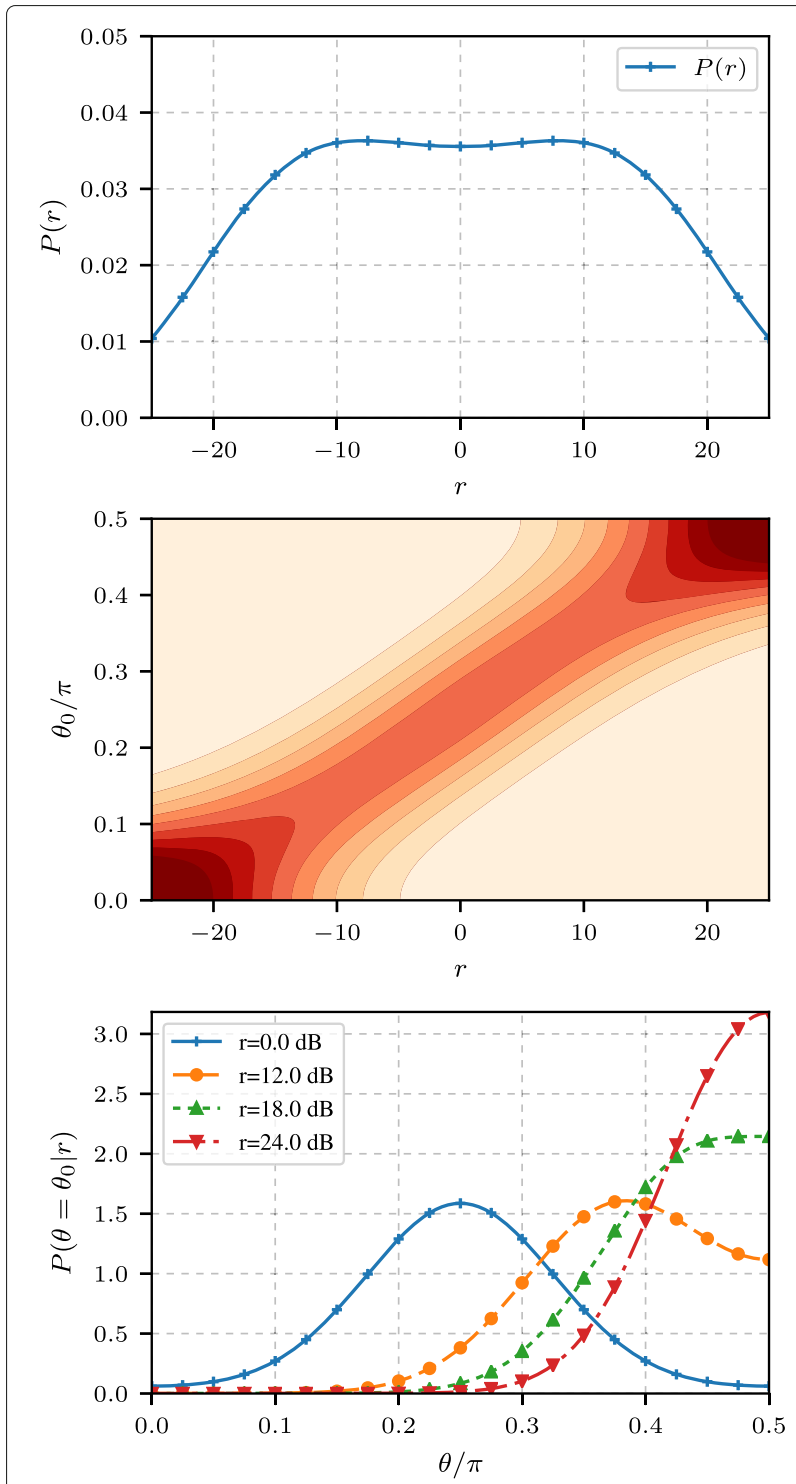

Fig. 6 Densities: marginal likelihood (a) and posterior density (b, c)

are some remarks on the measurement function $f=\Delta G(\phi)$. Measurement values $r$ are limited to $r \in[\min (\Delta G(\phi)), \max (\Delta G(\phi))]$. To transform arbitrary measurement values $r \in[-\infty, \infty]$ into parameter space, the definition of the inverted function $\Delta G^{-1}(r)$ function needs to be redefined:

$$
\Delta G^{-1}(r):=\left\{\begin{array}{lc}
0^{\circ} & \text { for } r<\min (\Delta G(\phi)) \\
90^{\circ} & \text { for } r>\max (\Delta G(\phi)) \\
\Delta G^{-1}(r) & \text { else }
\end{array}\right.
$$

With the posterior PDF, the MSE is computed by is defined by

$$
\operatorname{mse}(\hat{\phi})=\int_{-\pi / 2}^{\pi / 2} P(\phi \mid r)\left(\phi-\Delta G^{-1}(r)\right)^{2} \partial \phi .
$$


The results for the DOA root mean squared error (RMSE) are shown in Fig. 7. Remarkably, the variance of the MLE is bounded for all azimuth angles $\phi$. Furthermore, the MLE features a quite homogeneous distribution of DOA errors for all signal directions. In conclusion, there is no significant impact of the direction of the signal source on the variance of DOA estimates, though the MLE is biased.

\subsubsection{Position estimation errors}

Having derived the MSE for direction estimate, we can now evaluate the $2 \mathrm{D}$ position errors for a WSN featuring RSS-based DOA. As positioning errors are considered, the MLE position error bound describes an expectation on the MSE of the position estimator [21]. The expected MSE for a position estimate is denoted by

$$
\operatorname{mse}(\hat{\boldsymbol{x}})=E\left[(\hat{\mathbf{x}}-\mathbf{x})(\hat{\mathbf{x}}-\mathbf{x})^{\mathrm{T}}\right] .
$$

Recalling Eqs. (16) and (17), similar to the position CRLB, given by the error propagation law, the covariance matrix is expressed by [31]

$$
\left[H(\mathbf{x})^{-1}\right]_{i, j}=\left[\frac{\partial \boldsymbol{g}(\mathbf{x})}{\partial x_{i}}\right]^{\mathrm{T}} \frac{1}{\operatorname{mse}_{\theta}(\mathbf{x})} \boldsymbol{I}\left[\frac{\partial \boldsymbol{g}(\mathbf{x})}{\partial x_{j}}\right],
$$

where $\boldsymbol{I}$ denotes the identity matrix. Finally leading to

$$
H(\mathbf{x})^{-1}=\sum_{k}\left[\begin{array}{cc}
\frac{\Delta y_{k}^{2}}{\operatorname{mse}_{\theta}(\mathbf{x})\left\|\mathbf{x}-\mathbf{x}_{k}\right\|_{2}^{4}} & -\frac{\Delta x_{k} \cdot \Delta y_{k}}{\operatorname{mse}_{\theta}(\mathbf{x})\left\|\mathbf{x}-\mathbf{x}_{k}\right\|_{2}^{4}} \\
-\frac{\Delta x_{k} \cdot \Delta y_{k}}{\operatorname{mse}_{\theta}(\mathbf{x})\left\|\mathbf{x}-\mathbf{x}_{k}\right\|_{2}^{4}} & \frac{\Delta x_{k}^{2}}{\operatorname{mse}_{\theta}(\mathbf{x})\left\|\mathbf{x}-\mathbf{x}_{k}\right\|_{2}^{4}}
\end{array}\right]
$$

for location information obtained from RSS-based DOA measurements, with $\|\mathbf{x}\|_{2}$ denoting the euclidean norm.

In its compressed form, the position estimation error is expressed by

$$
\operatorname{mse}_{\mathbf{x}}(\mathbf{x})=\operatorname{tr}(\boldsymbol{H}(\mathbf{x}))
$$

for DOA-based network localization when applying ML estimation.

\subsubsection{Comparison of network topologies}

The derivation of the position error bound for RSSbased network localization is now utilized to compare the performance of different network topologies for the $\mathrm{ML}$ approach. The DOA estimation error mse $_{\theta}$ is computed according to Eq. (33). Mean square position errors are evaluated for three different WSN topologies. The locating networks consist of four nodes and have a node spacing of $50 \mathrm{~m}$. Receive antenna are being rotated by $0^{\circ}$, $30^{\circ}$, and $45^{\circ}$ for the examined network topologies 1,2 , and 3, respectively. Sensor nodes have been arranged in quadratic shape. All networks have been examined for an area of interest of $50 \times 50 \mathrm{~m}$.

As elaborated in Section 3, the network topology, more specifically the rotation of the receive antenna arrays, is a crucial parameter in terms of position estimation errors. This holds true for the MVUE. However, results for the ML approach are different. In Fig. 8, the resulting position estimation errors for all the three networks are given. For the given rotation angles of the antenna arrays, there is no substantial difference in average RMSE. Results are shown in Table 2. The average RMSE for the MLE is constant for different node orientation angles, whereas the average RMSE significantly depends on the position of the tracked object for the MVUE. In conclusion, the rotation of the nodes is negligible for the design of the WSN when considering the MLE. These results are in contrast to those for the MVUE, where the RMSE is highly dependent of the node orientation.

\subsubsection{Results and discussion}

With the MLE, we have shown that the topology of the locating WSN does not have a significant impact on the positioning. These findings are in contrast to the results of the CRLB for the MVUE. For snapshot localization, the MLE should be preferred since it features smaller RMSEs in any case. Yet, unbiasedness comes at the cost of an

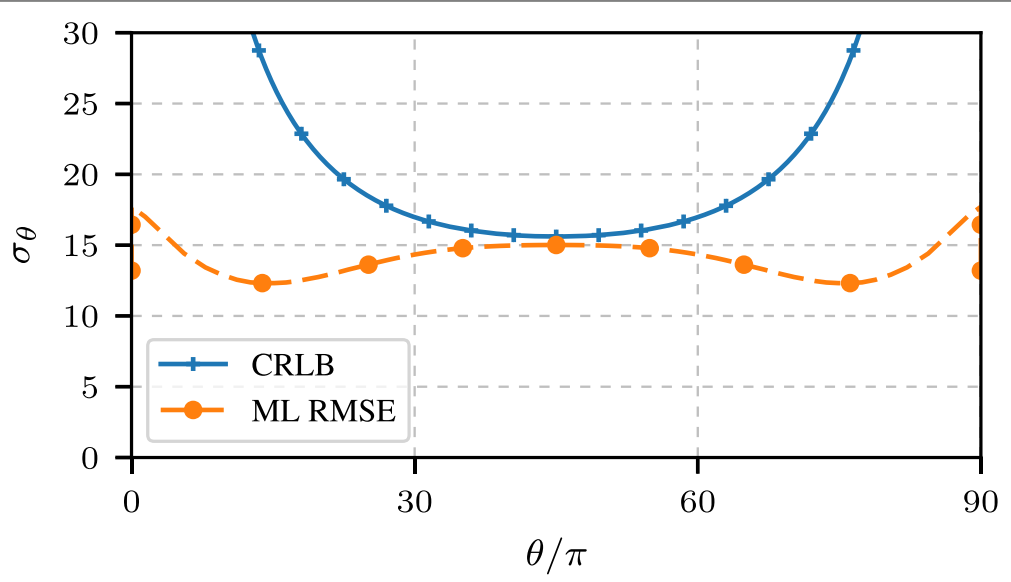

Fig. 7 CRLB compared to ML RMSE. With the ML estimator, the RMSE is significantly reduced compared to the MVUE at the cost of being biased 


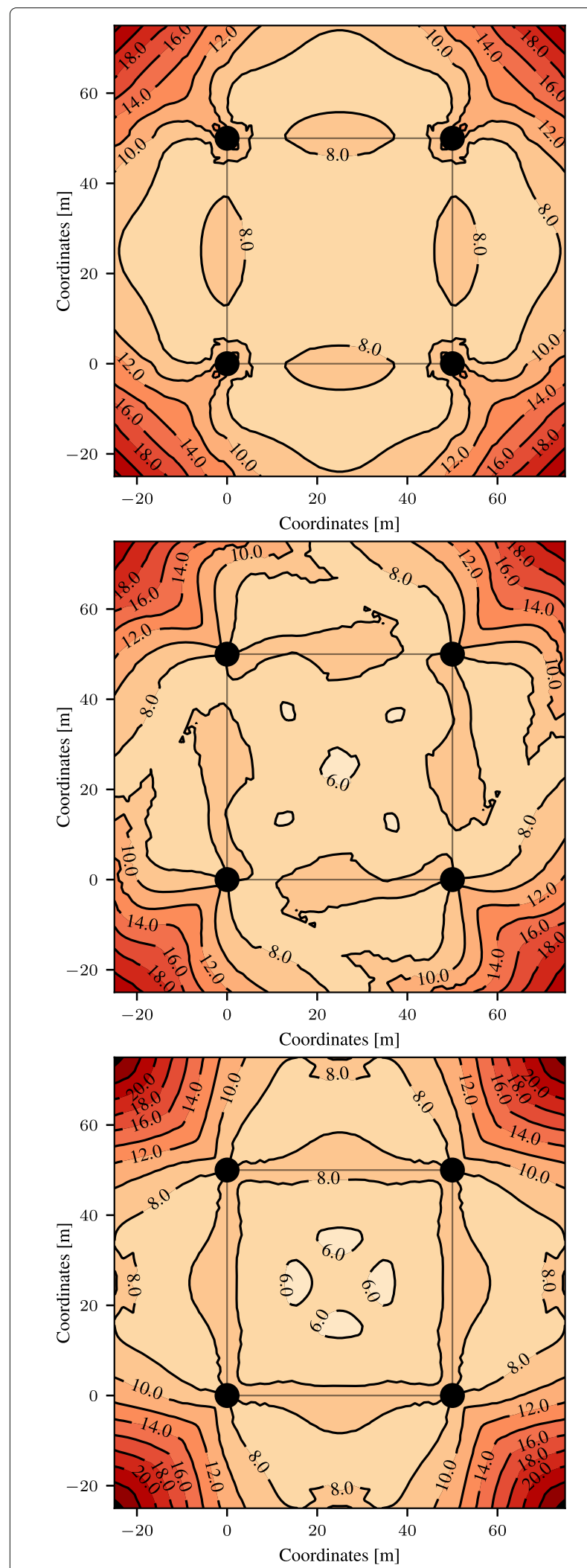

Fig. 8 Position RMSE for different rotation of sensor nodes. a Rotation: $0^{\circ}$. b Rotation: $30^{\circ}$. c Rotation: 45
Table 2 Comparison of networks and estimators

\begin{tabular}{llll}
\hline & \multicolumn{3}{l}{ Networks } \\
\cline { 2 - 4 } Rotation angle & $0^{\circ}$ & $30^{\circ}$ & $45^{\circ}$ \\
\hline Avg. RMSE for MLE & 7.19 & 7.26 & 7.23 \\
Avg. RMSE for MVUE & 10.13 & 8.61 & 12.89 \\
\hline
\end{tabular}

increasing variance resulting in a larger MSE for positioning (cf. Fig. 9). Considering recursive filtering, one might prefer the MVUE over the MLE as the higher variance can be averaged out applying motion models. But still, the analysis of the MLE lacks the capability of considering ambiguous measurements. In the next section, we introduce information-theoretic measures to provide an insight into the gain of information retrieved from a single measurement obtained from a WSN.

\section{Information-theoretic localization performance metrics}

Despite the success of information theory in communications, information theory has not caught much attention in localization and navigation in the past, except for [32, 33]. Recently, there has been a revival of information theory in many fields. Information-theoretic measures, like the mutual information, have been utilized to optimize MIMO radar waveforms [11]. The loss of information due to sub-Nyquist sampling has been determined applying information theory [12, 13]. Currently, there are big advances in the field of compressive sensing. Lately, fundamental limits in compressed sensing [14] and bounds for kernel-based time delay estimation [15] are derived.

However, classical estimation theory is still in the focus of performance analysis today. Nevertheless, the CRLB is a local measure for the variance of an estimator. Considering a uniform linear antenna array, the CRLB [34] states that

$$
\operatorname{var}(\hat{\phi}) \propto \frac{1}{d^{2}}
$$

where $d$ is the distance between the antenna elements. In consequence, distance between elements should be maximized. However, ambiguities arise for a spacing of more than $\lambda / 2$. These need to be considered in the design of sensor nodes. As the CRLB is a local measure, it is blind for ambiguities. Hence, the CRLB is only applicable when further constraints are introduced. Information theory, in contrast to the CRLB, has a unified view on the information gained from a set of sensor measurements considering precision and ambiguities. 

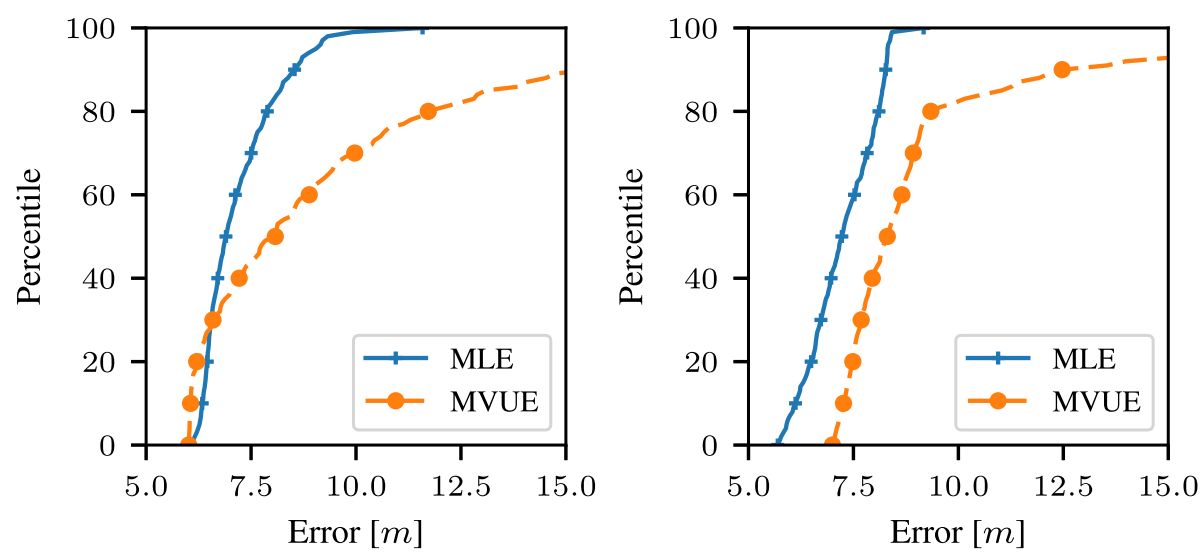

Fig. 9 Position errors (percentiles). On the left for the WSN with node rotation $0^{\circ}$, on the right for a rotation of $45^{\circ}$

The entropy $H(X)$ of a discrete random variable $X$ is defined by the expectation value of its information given by $[35,36]$

$$
H(X)=-\sum_{x \in E} P(X=x) \log (P(X=x)) .
$$

Maximum entropy is achieved by a uniform probability distribution for a given number of discrete values. Physical quantities, as in network localization, generally have no discrete values. Measurement and state variables are continuous. Therefore, the limit of the entropy from discrete values to a continuous range would diverge. To circumvent this, the differential entropy is defined [36]

$$
h(X)=-\int_{E} p_{X}(x) \log \left(p_{X}(x)\right) \mathrm{d} x .
$$

In contrast to entropy, the differential entropy can be negative and the differential entropy of a constant diverges. For an infinite interval (i.e., $x \in]-\infty,+\infty[$ ) and fixed variance, the normal distribution maximizes the differential entropy, whereas for a fixed interval (i.e., $x \in[a, b]$ ), the uniform distribution is maximum.

For illustration, we consider some well-known distributions: uniform, normal, and von Mises distribution (Table 3). The von Mises distribution [37] is defined over $\phi \in[-\pi ;+\pi]$

$$
p(\theta)=\frac{e^{\kappa \cos (m \cdot(\theta-\mu))}}{2 \pi \mathrm{I}_{0}(\kappa)},
$$

where $\mu$ is the mean value and $\kappa$ defines the concentration of the distribution. $I_{0}$ is the modified Bessel function

Table 3 Entropy of some important distributions

\begin{tabular}{lll}
\hline Distribution & PDF & Entropy \\
\hline Uniform [38] & $p(\theta)=\frac{1}{b-a}$ & $h=\operatorname{ld}(b-a)$ \\
Normal [38] & $p(\theta)=\frac{e^{\left(-\theta / 2 \sigma^{2}\right)}}{\sqrt{2 \pi \sigma^{2}}}$ & $h=\frac{1}{2} \operatorname{ld}\left(2 \pi e \sigma^{2}\right)$ \\
von Mises [39] & $p(\theta)=\frac{e^{\kappa \cos (\theta)}}{2 \pi l_{0}(\kappa)}$ & $h=-\kappa \frac{l_{1}(\kappa)}{I_{0}(\kappa)}+\operatorname{ld}\left(2 \pi I_{0}(\kappa)\right)$ \\
\hline
\end{tabular}

of order $0 . m$ denotes the number of modes of the distribution and is understood as some scaling factor for the von Mises distribution. The concentration is reciprocal to dispersion. Hence, $\frac{1}{\kappa}$ is comparable to the variance. Large concentration implies low variance and vice-versa. For $\kappa \gg 0$, the von Mises distribution approaches the normal distribution with $\sigma^{2}=1 / \kappa$. Therefore, it is a good approximation of the wrapped normal distribution. Its differential entropy is given by [37]

$$
h(\theta)=\log \left(2 \pi I_{0}(\kappa)\right)-\frac{\kappa I_{1}(\kappa)}{\log (e) I_{0}(\kappa)},
$$

where $I_{1}$ is the first-order modified Bessel function.

In Fig. 10, von Mises distributions (blue and orange) with different $\kappa$ are depicted. It can be seen that with rising $\kappa$, i.e., higher concentration around the mean value, entropy, denoted by $h$, declines. Actually, the standard deviation of the blue density is three times larger than the deviation of orange density. $\kappa=0$ (uniform) yields maximum entropy. Furthermore, a repeated and scaled version (green line) of the von Mises distribution is sketched in Fig. 10. The green distribution has locally the same standard deviation as the orange one. Thus, in terms of the CRLB, both, the orange and the green, yield the same precision. However, when considering the total information gained from the measurement, the blue and the green distribution are comparable as they feature the same entropy. Actually, the multimodal PDF can be seen as ambiguous measurements. Even though a single lobe of a multimodal distribution features a small variance on a local scale, the entropy is the same compared to a broader lobe of a unimodal distribution.

To conclude, entropy does not give a hint if ambiguities are apparent. Neither does the CRLB. The variance inferred from the CRLB is a local measure. Hence, it does not give any information on the modality of the distribution. The mutual information only states how much information was gained by a measurement regardless if 


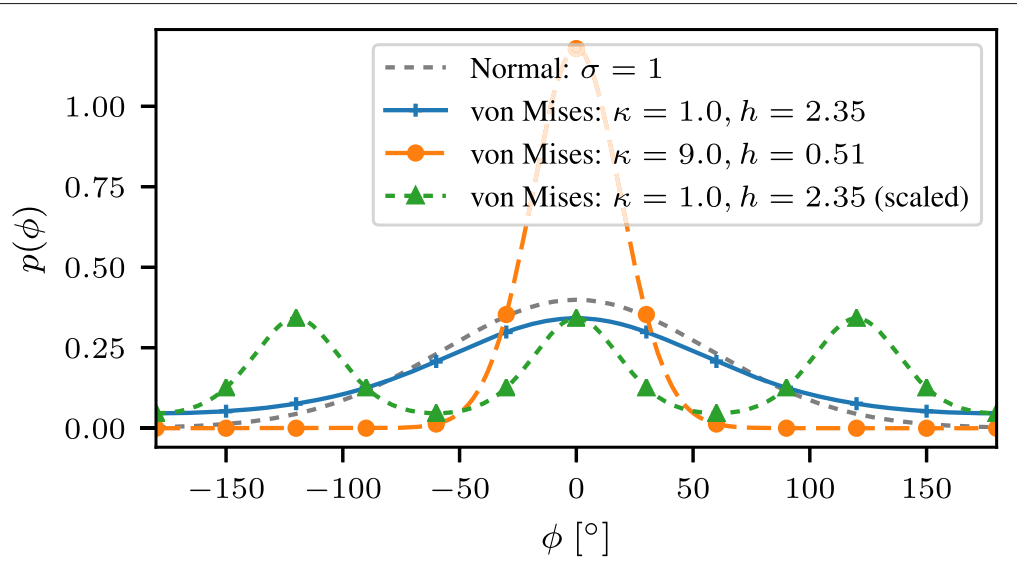

Fig. 10 PDFs for different von Mises distributions. The three times repeated version with $\kappa=1$ (green) has the same lobe width as the von Mises distribution with $\kappa=9$ (orange) but matches the entropy of the von Mises distribution with $\kappa=1$ (blue)

the resulting PDF is a broad unimodal Gaussian or a set of multiple sharp Gaussian distributions. Therefore, the CRLB is a local measure and the entropy quantifies the overall information gain. In fact, both approaches lead to the same result if the underlying distribution is a unimodal Gaussian.

\section{Network topology optimization}

In this section, we utilize the mutual information as a criterion for network topology optimization. For the illustration, we use a simple measurement model for the DOA estimation. The bearing measurements are assumed to be impaired by AWGN and ambiguous. Furthermore, we define the variance of the DOA measurements to be independent of the direction of the impinging signal. As the estimated angle $\hat{\phi}$ is limited to the interval $\phi \in[-\pi ;+\pi]$, its PDF is to be described by a wrapped normal distribution. Commonly, the wrapped normal distribution is approximated by the von Mises distribution.

For the rest of the section, we consider two different measurement likelihoods:

1 The ambiguous DOA sensor:

Provides multimodal DOA measurements with constant variance. Ambiguities arise due to point and axial symmetry exactly the same way as they arise for the antenna considered in the BATS project.

2 The unambiguous DOA sensor:

Provides unimodal DOA measurements with constant variance over angles. This unambiguous sensor, that features locally the same variance as the ambiguous one, is used as a reference. The ambiguous sensor is benchmarked against the unambiguous sensor.

Considering the BATS DOA sensor, the likelihood is state as

$$
p(\phi)=\frac{1}{2} \frac{e^{\kappa \cos (2 \phi)}}{2 \pi I_{0}(\kappa)}+\frac{1}{2} \frac{e^{\kappa \cos (-2 \phi)}}{2 \pi I_{0}(\kappa)} .
$$

The likelihood of the unambiguous DOA sensor is given by

$$
p(\phi)=\frac{e^{\kappa \cos (\phi)}}{2 \pi I_{0}(\kappa)} .
$$

The PDFs for both of the estimators are depicted in Fig. 11. For reference, the entropy of a uniform $\mathrm{U}(-\pi, \pi)$ is $h=$ 2.65. It can be easily seen that, considering the entropy of both sensor types, the unambiguous sensor has a significantly lower entropy. Thus, the unambiguous sensor provides more information. This is quite obvious, as the unambiguous sensor perfectly resolves the ambiguities arising in the BATS DOA sensor. On the other hand, with respect to the CRLB, both yield the same performance.

\subsection{A comparison of network topologies}

The two considered DOA sensors are examined in two network localization scenarios. The impact of ambiguities in BATS DOA sensor on the localization performance is examined in the following. Therefore, the BATS DOA sensor is compared to an unambiguous sensor with same local precision as described above. It can be shown that even for a small number of sensor nodes, the ambiguities arising on sensor level can be almost completely resolved on position level.

Network localization scenario 1 consists of two sensor nodes placed at $[0,25]$ and $[30,25]$. The object to be tracked resides at $[25,35]$. For network scenario 1 , the likelihoods for the unambiguous DOA sensor and the BATS DOA sensor are depicted in Fig. 12. The depicted likelihoods involve all sensor nodes, i.e., the shown likelihood is the product of the likelihoods of the individual sensors. The likelihood is centered around the true position for the unambiguous bearing measurement, whereas in case of the ambiguous sensor, the likelihood PDF features four 


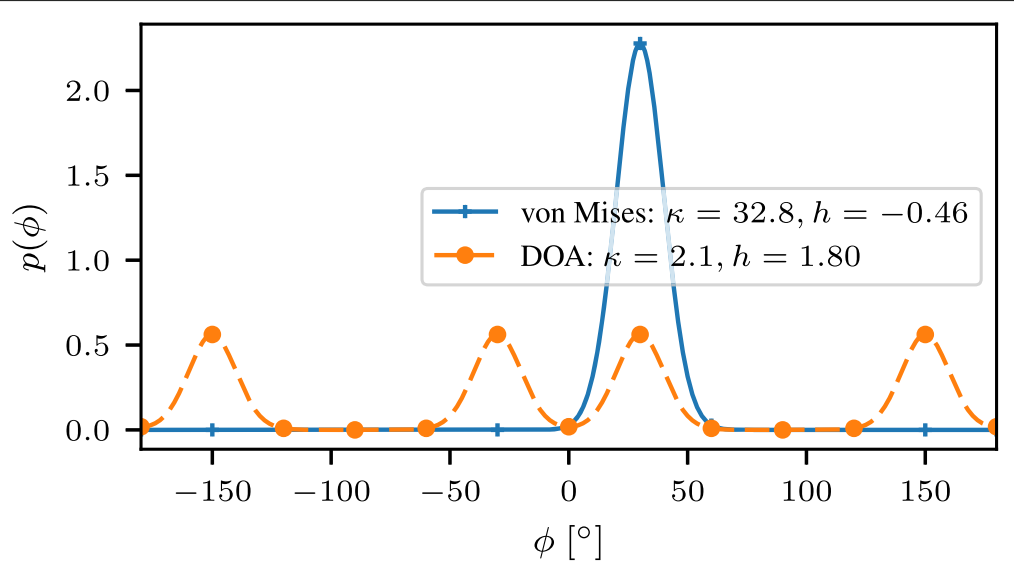

Fig. 11 PDFs for the unambiguous and the ambiguous DOA sensor for $\phi=30^{\circ}$ with a standard deviation of $\sigma=15^{\circ}$ sketched in blue and orange, respectively

modes. In consequence, a tracking filter would have to be capable of propagating multiple hypotheses. Hence, in such a case, particle filters would have to be used instead of basic Kalman filters.

For the evaluation, a single snapshot measurement is considered. The prior information available is compared to entropy after the measurement. If there is no prior information available, we assume a non-informative prior, which relates to a $2 \mathrm{D}$ uniform distribution. Over an area of interest of $70 \times 70 \mathrm{~m}$, the entropy of a uniform is $h=12.26$. Entropy is computed for the networks for the ambiguous DOA sensor and the unambiguous one. The information gain of such a set of DOA measurements is given by the mutual information. Mutual information is computed from entropy and conditional entropy

$$
I(\mathbf{x} ; \phi)=h(\mathbf{x})-h(\mathbf{x} \mid \phi),
$$

For the network localization scenario 1, the corresponding entropy and mutual information is given in Table 4. As expected, ambiguous DOA has a larger entropy than unambiguous DOA.

Now, two additional nodes are placed at $[0,50]$ and $[50,0]$ for the second network localization scenario. The true position of the object is, as before, at $[25,35]$. Likelihoods for the ambiguous and unambiguous DOA sensor are illustrated in Fig. 13. As stated before, all sensors depicted in the figure are involved in the computation of the likelihood shown Fig. 13. It can be clearly seen that the true position becomes the dominant hypothesis for the ambiguous bearing sensor (cf. Fig. 12). On the other hand, for the unambiguous sensor, the position PDF becomes more diffuse. When more sensors are used, the impact of the ambiguities gets less important. Furthermore, the gain in information for additional sensors is not very significant when unambiguous DOA sensors are utilized. Effectively, additional sensors do not provide as much information as the first two sensors (cf. Table 4). In conclusion, for overdetermined sensor networks, ambiguous sensors are an option as the impact of ambiguities becomes negligible when a larger number of sensors is deployed. Additionally, accepting ambiguities commonly results in higher precision for the individual modes (cf. DOA estimation applying linear antenna arrays).

\subsection{From entropy to localization precision}

One disadvantage of the mutual information is that it is not as intuitive as a standard deviation. Thus, we are interested in computing variance from the entropy. The relation for variance and entropy assuming a normal distribution is expressed by

$$
h=\frac{1}{2} \operatorname{ld}(2 \pi e|\Sigma|) .
$$

The variance of a symmetric $2 \mathrm{D}$ normal distribution (i.e., $|\Sigma|=\sigma^{2 k}$, with $\Sigma$ being of size $\left.k \times k\right)$ is computed as follows

$$
\sigma^{2}=\frac{\sqrt[k]{2^{2 h}}}{2 \pi e}
$$

for a given entropy $h$. Nevertheless, it has to be noted that this expression is only a good approximation when standard deviation is small compared to the size of the area of interest. Aside from that, the complete mutual information is assumed to arise from a single 2D Gaussian. For a multimodal distribution, the entropy inferred variance is larger than the variance of the individual modes as the above stated transformation is only valid for unimodal Gaussian distributions.

In Table 4, standard deviation for the two example networks is given for the unambiguous and ambiguous DOA sensor, respectively. The standard deviation computed under the assumption of a unimodal 2D Gaussian 

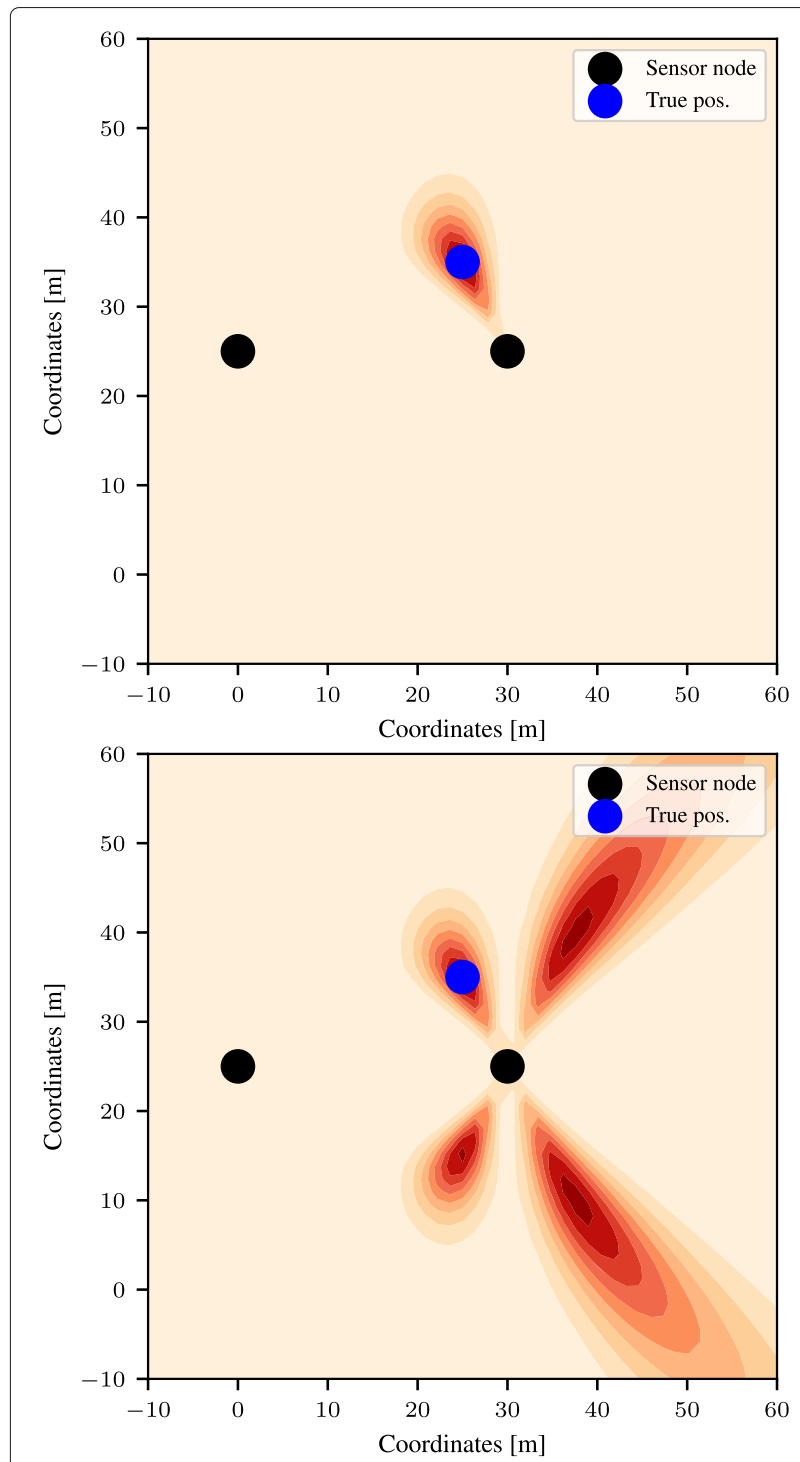

Fig. 12 Likelihoods for example network localization scenario 1 for the unambiguous DOA sensor and the BATS DOA sensor. True position of the tracked object is at $[25,35]$. a Unambiguous bearing. $\mathbf{b}$ Ambiguous bearing
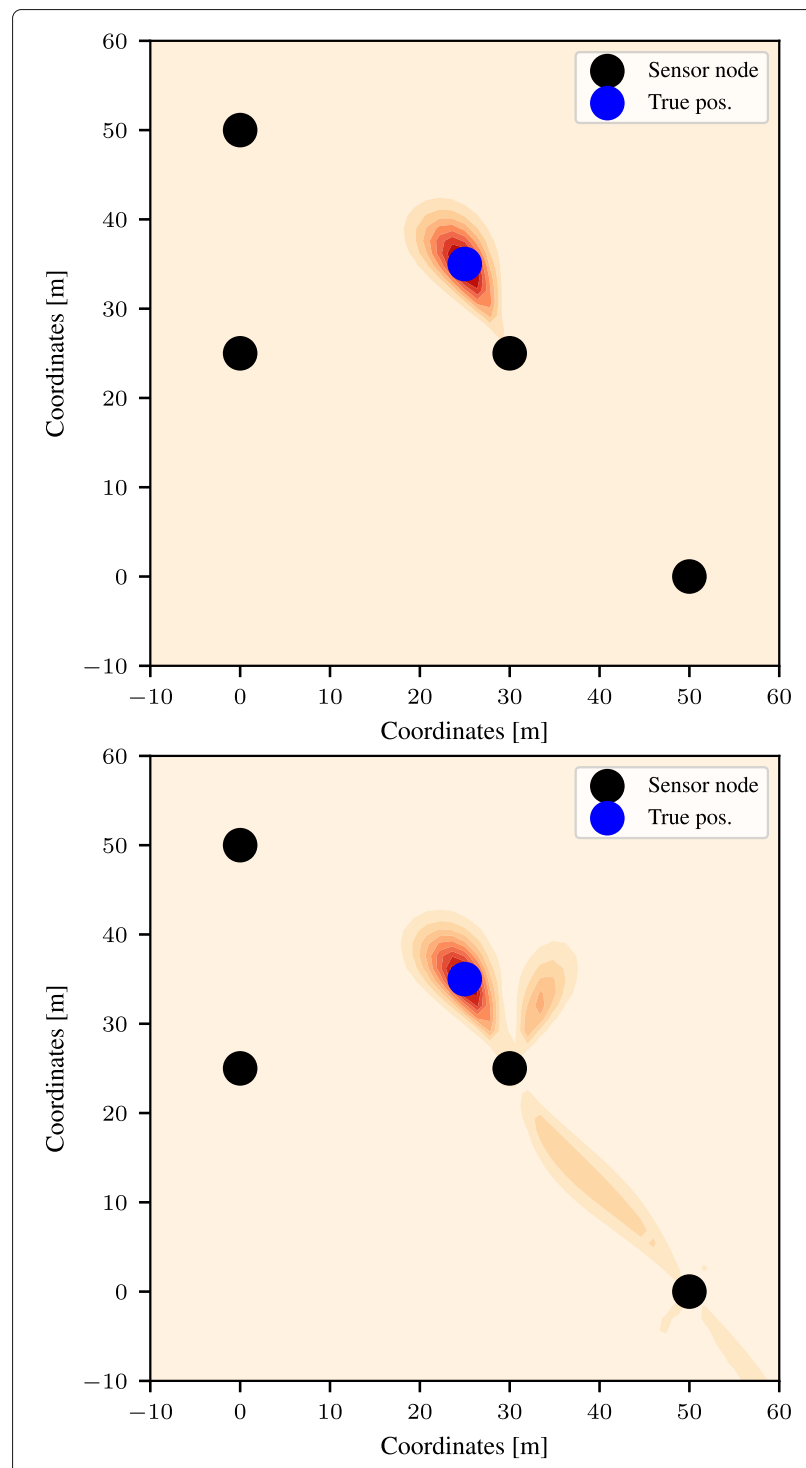

Fig. 13 Likelihoods for example network localization scenario 2 for the unambiguous DOA sensor and the BATS DOA sensor. True position of the tracked object is at $[25,35]$. a Unambiguous bearing. b Ambiguous bearing
Table 4 Entropy and mutual information of different DOA networks

\begin{tabular}{|c|c|c|c|}
\hline Sensor & Entropy & Mutual inf. & Equiv. dev \\
\hline \multicolumn{4}{|l|}{ Network 1} \\
\hline Unambiguous & 8.24 bit & 4.02 bit & $4.21 \mathrm{~m}$ \\
\hline BATS DOA & 9.91 bit & 2.35 bit & $7.50 \mathrm{~m}$ \\
\hline \multicolumn{4}{|l|}{ Network 2} \\
\hline Unambiguous & 7.16 bit & 5.10 bit & $2.89 \mathrm{~m}$ \\
\hline BATS DOA & 8.27 bit & 3.99 bit & $4.25 \mathrm{~m}$ \\
\hline
\end{tabular}

proofs the conclusions drawn from Figs. 12 and 13. The impact of ambiguities is less important when using more sensor nodes. The total information gain is negligible for additional sensors in the case of unambiguous DOA.

\subsection{Mutual information as a network design tool}

In the section above, we have shown that the use of additional sensor nodes may mitigate the impact of ambiguities. Hence, it is desirable to compare different network topologies considering different sensor types. For the analysis, we consider the unambiguous and ambiguous DOA sensors introduced above. The design question 
to be answered is: How many nodes are required to realize a certain gain of information about the position of an object? For the sake of simplicity, we assume that all nodes are place equidistantly on a circle with fixed radius. Hence, the only parameters are the number of nodes and the sensor type.

Examples of network configurations for 2 and 4 nodes are given in Fig. 14a and b, respectively. For an area of interest of $70 \times 70 \mathrm{~m}$, the conditional entropy is computed for the PDF after the network localization measurement. We assume no prior information is available before the measurement. Mutual information is computed by (45) for all network topologies and sensor types under test. A comparison of the unambiguous and ambiguous DOA sensor is depicted in Fig. 15. It can be easily seen that utilizing an additional number of two sensor nodes allows to mitigate the negative impact of the ambiguities for the ambiguous DOA sensor.

Mutual information provides a tool to compare different network topologies including multimodal measurement PDFs, whereas the classic CRLB fails in this case. CRLB analysis would provide exactly the same results for the unambiguous and ambiguous DOA sensor, which is quite misleading. In contrast, information theory allows for exact quantification of the impact of ambiguities on the information that is gain on an object's position. With the use of this design tool, based on mutual information, engineers are able to design the most effective networks for localization problems.

\section{Conclusion}

In this paper, different performance metrics have been analyzed and applied in the context of RSS-based direction finding. The first of those metrics is the CRLB. It has been shown that the CRLB strongly depends on the direction of the impinging signal for power-based DOA estimation. Due to the nature of the DOA measurement function, the CRLB diverges for some angles. Hence, the MVUE is not a reasonable estimator in the case of RSSbased DOA.

Secondly, a performance metric has been derived for the MLE evaluating the posterior density for RSS-based DOA estimates. In this case, DOA estimation variance is nearly constant for all signal directions. Hence, the variance in position estimation is less dependent on the actual position of the tracked object. Furthermore, the MSE for the MLE is significantly smaller than for the MVUE. However, both the CRLB and the error bound for the MLE lack the capability of handling ambiguities.

In order to account for ambiguities, we have proposed a novel approach to assess the performance of localization systems that feature sensors with multimodal measurement likelihoods. Information-theoretic measures are
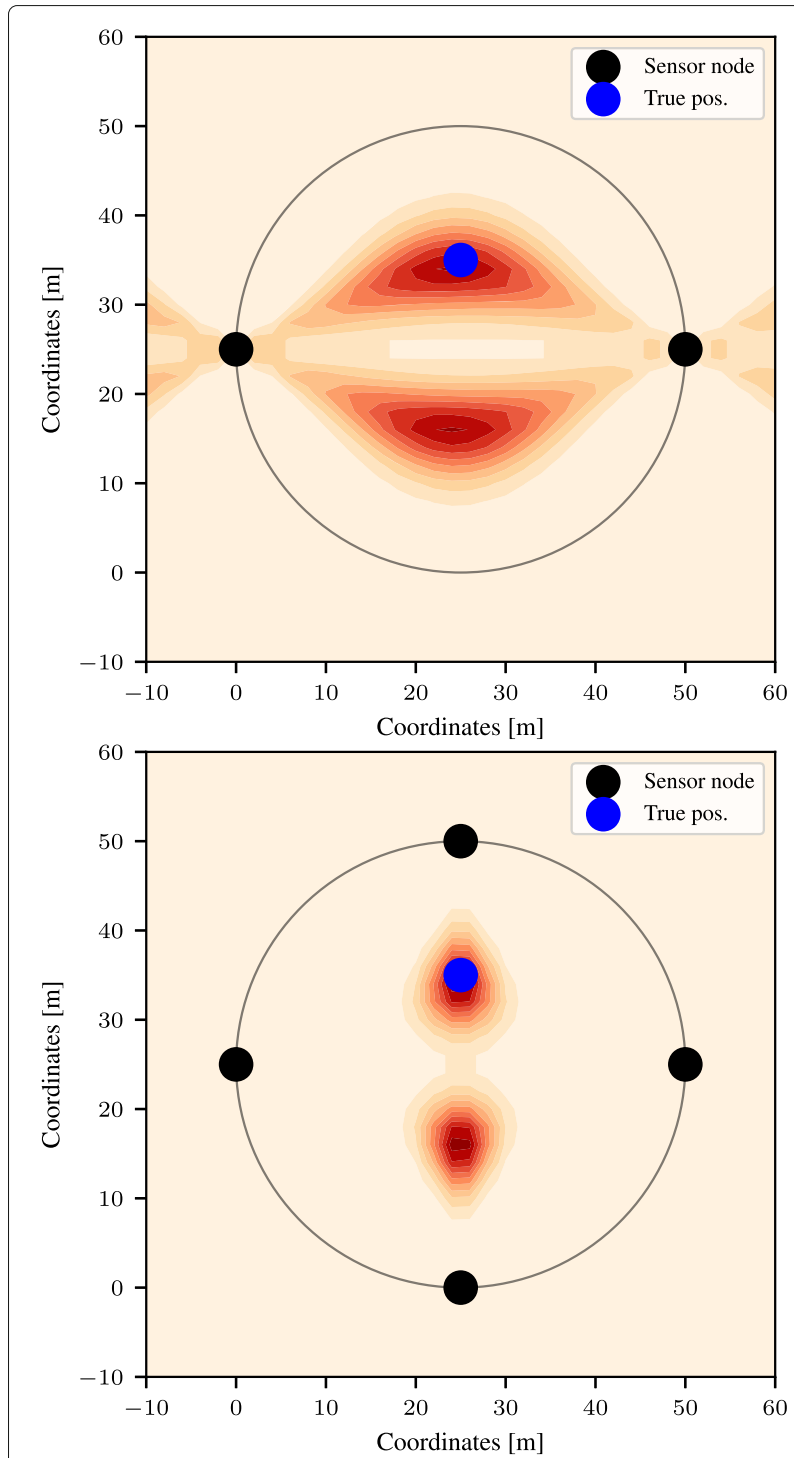

Fig. 14 Likelihoods for different number of nodes. All nodes are equidistantly placed on a circle as indicated in the figure. True position of the tracked object is at $[25,35]$. a Ambiguous bearing: 2 nodes. b Ambiguous bearing: 4 nodes

utilized to quantify the information gain of a set of measurements from multiple sensor nodes. With this unified approach, all aspects, i.e., local precision and multimodality, can be captured with a single measure: the mutual information. The presented information-theoretic approach allows for unified optimization of location sensors and localization networks in effective way maximizing the mutual information. Moreover, mutual information could be used to quantify the loss when utilizing sub-optimal estimators and for performance assessment of recursive filters. In total, information-theoretic metrics provide a holistic view on the performance of tracking systems. 


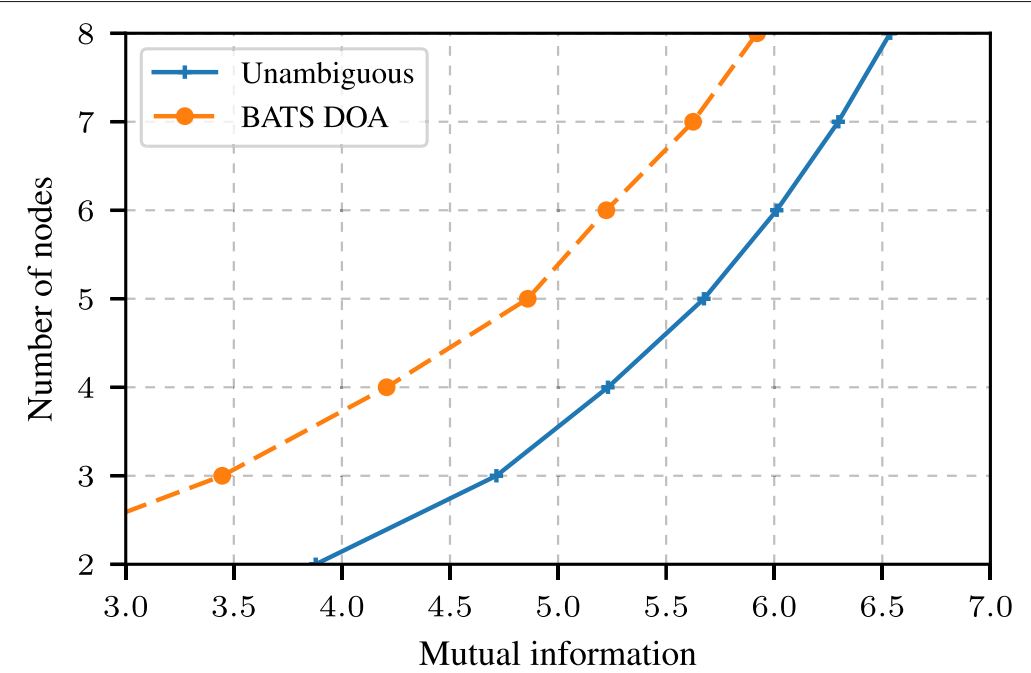

Fig. 15 Required number of sensor nodes to realize a requested amount of information gain by a network localization measurement

\section{Endnote}

${ }^{1}$ Dynamic Adaptable Applications for Bats Tracking by Embedded Communicating Systems, http://www.forbats.org/

\section{Abbreviations}

AWGN: Additive white Gaussian noise; CRLB: Cramér-Rao lower bound; DOA: Direction-of-arrival; FIM: Fisher information matrix; ML: Maximum likelihood; MLE: Maximum likelihood estimator; MSE: Mean squared error; MVUE: Minimum variance unbiased estimator; PDF: Probability density function; RMSE: Root mean squared error; RSS: Received signal strength; RV: Random variable; TDOA: Time-difference-of-arrival; WSN: Wireless sensor network

\section{Funding}

This work is funded by the German Science Foundation DFG grant FOR 1508, Research Unit BATS.

\section{Availability of data and materials}

All data are based on simulations. Scripts for data simulation and evaluation will be made available on demand for the review. Simulation data will be made available on the institutional website when the manuscript is published.

\section{Authors' contributions}

The main contribution to this manuscript is by TN. MH contributed to the antenna design and development as well as the CRLB in RSS-based DOA. JT developed the initial ideas and the proposal of the BATS project. He continuously supported the project work with his expertise in navigation and localization. Furthermore, he did a thorough revision of the content published. All authors read and approved the final manuscript.

\section{Authors' information}

Thorsten Nowak received his diploma in engineering from the University of Ulm, Germany, in 2009, and is currently working toward the Ph.D. degree at Friedrich-Alexander-Universität Erlangen-Nürnberg (FAU). In 2008, he joined Fraunhofer Institute for Integrated Circuits (IIS), where he was a research assistant at the Locating \& Communication Systems Department within the Sensor Fusion \& Event Processing Group. His focus was on multipath mitigation techniques, multisensor data fusion, localization systems, and RFID. Since 2013, he is with Friedrich-Alexander-Universität Erlangen-Nürnberg (FAU), working for the Department of Electrical, Electronic and Communication Engineering. He is a research assistant at the Insitute of Information Technology (Communication Electronics) and is involved in the Research Unit BATS-Dynamic Adaptable Applications for Bats Tracking by Embedded Communicating Systems funded by the German Research Foundation (DFG).
Multipath: He regularly serves in the program committee of the IEEE ICC and is a reviewer for the IEEE Systems Magazine, the International Journal of Microwave and Wireless Technologies, and the Elsevier Journals of Adhoc Networks and Computer Communications.

Markus Hartmann received his diploma in engineering, in 2009, from the University of Applied Sciences Amberg-Weiden, Germany, and his master of engineering, in 2011, from the University of Applied Sciences Coburg, Germany. In 2010, he joined Fraunhofer Institute for Integrated Circuits (IIS), where he was a research assistant at the Locating and Communication Systems Department within the RFID and Radio System group. His research focus was on inductive low-frequency localization technologies for a goal line decision system. He is currently working toward the Ph.D. degree at Friedrich-Alexander-University Erlangen-Nürnberg (FAU) in the area of localization based on phase and signal strength informations. His main focus is now on the performance evaluation and error analysis of field strength based localization approaches.

Jörn Thielecke received a PhD (Dr.-Ing.) from the

Friedrich-Alexander-Universität Erlangen-Nürnberg (FAU), Germany. In 1991, he joined Philips Kommunikations Industrie AG and in 1997 Ericsson leading a research group on radio access for cellular mobile radio communications. He was responsible for the Department of Communications at the Research Establishment for Applied Science (FGAN) from 2003 until 2004. Now, he is professor at FAU focusing on localization and navigation systems.

\section{Competing interests}

The authors declare that they have no competing interests.

\section{Publisher's Note}

Springer Nature remains neutral with regard to jurisdictional claims in published maps and institutional affiliations.

\section{Received: 19 December 2017 Accepted: 20 June 2018}

Published online: 18 July 2018

\section{References}

1. I Parvez, F Abdul, Al Sarwat, in 2016 IEEE Green Technologies Conference (GreenTech). A location based key management system for advanced metering infrastructure of smart grid, (2016), pp. 62-67. https://doi.org/ 10.1109/GreenTech.2016.19

2. I lala, M Ouadou, D Aboutajdine, O Zytoune, in 2017 International Conference on Advanced Technologies for Signal and Image Processing (ATSIP). Energy based collision avoidance at the mac layer for wireless sensor network, (2017), pp. 1-5. https://doi.org/10.1109/ATSIP.2017. 8075611 
3. R Kays, S Tilak, M Crofoot, T Fountain, D Obando, A Ortega, F Kuemmeth, J Mandel, G Swenson, T Lambert, B Hirsch, M Wikelski, Tracking animal location and activity with an automated radio telemetry system in a tropical rainforest. Comput. J. 54(12), 1931-1948 (2011). https://doi.org/ 10.1093/comjnl/bxr072

4. J Hightower, G Borriello, Location systems for ubiquitous computing. Computer. 34(8), 57-66 (2001). https://doi.org/10.1109/2.940014

5. M Vossiek, L Wiebking, P Gulden, J Wieghardt, C Hoffmann, P Heide, Wireless local positioning. IEEE Microw. Mag. 4(4), 77-86 (2003). https:// doi.org/10.1109/MMW.2003.1266069

6. MZ Win, Y Shen, H Wymeersch, in Spread Spectrum Techniques and Applications, 2008. ISSSTA '08. IEEE 10th International Symposium On. On the position error bound in cooperative networks: a geometric approach, (2008), pp. 637-643. https://doi.org/10.1109/ISSSTA.2008.125

7. $\mathrm{KYu}, 3-\mathrm{D}$ localization error analysis in wireless networks. IEEE Trans. Wirel. Commun. 6(10), 3472-3481 (2007). https://doi.org/10.1109/TWC.2007. 05596

8. Y Shen, MZ Win, Fundamental limits of wideband localization_Part I: a general framework. IEEE Trans. Inf. Theory. 56(10), 4956-4980 (2010). https://doi.org/10.1109/TIT.2010.2060110

9. D Dardari, A Conti, U Ferner, A Giorgetti, MZ Win, Ranging with ultrawide bandwidth signals in multipath environments. Proc. IEEE. 97(2), 404-426 (2009). https://doi.org/10.1109/JPROC.2008.2008846

10. C Chang, A Sahai, Cramér-Rao-type bounds for localization. EURASIP J. Adv. Signal Process. 2006(1), 094287 (2006). https://doi.org/10.1155/ASP/ 2006/94287

11. Y Yang, RS Blum, MIMO radar waveform design based on mutual information and minimum mean-square error estimation. IEEE Trans. Aerosp. Electron. Syst. 43(1), 330-343 (2007). https://doi.org/10.1109/ TAES.2007.357137

12. Y Chen, AJ Goldsmith, YC Eldar, On the minimax capacity loss under sub-nyquist universal sampling. IEEE Trans. Inf. Theory. 63(6), 3348-3367 (2017). https://doi.org/10.1109/TIT.2017.2695541

13. A Kipnis, AJ Goldsmith, YC Eldar, in 2015 IEEE Information Theory Workshop (ITW). Sub-NYQUIST sampling achieves optimal rate-distortion, (2015), pp. 1-5. https://doi.org/10.1109//TW.2015.7133113

14. A Kipnis, G Reeves, YC Eldar, AJ Goldsmith, in Information Theory (ISIT), 2017 IEEE International Symposium On. Fundamental limits of compressed sensing under optimal quantization, (2017)

15. Y Gu, NA Goodman, Information-theoretic compressive sensing kernel optimization and Bayesian Cramer Rao bound for time delay estimation. IEEE Trans. Signal Process. 65(17), 4525-4537 (2017). https://doi.org/10. 1109/TSP.2017.2706187

16. M Hartmann, O Pfadenhauer, L Patino-Studencka, H-M Troeger, A Heuberger, J Thielecke, in Proceedings of the ION 2015 Pacific PNT Meeting. Antenna pattern optimization for a rssi-based direction of arrival localization system, (2015), pp. 429-433

17. THe, C Huang, BM Blum, JA Stankovic, T Abdelzaher, in Proceedings of the 9th Annual International Conference on Mobile Computing and Networking. Range-free localization schemes for large scale sensor networks (ACM, 2003), pp. 81-95

18. F Dressler, S Ripperger, M Hierold, T Nowak, C Eibel, B Cassens, F Mayer, K Meyer-Wegener, A Koelpin, From radio telemetry to ultra-low power sensor networks-tracking bats in the wild. IEEE Commun. Mag. 54(1), 129-135 (2016). https://doi.org/10.1109/MCOM.2016.7378438

19. M Hartmann, T Nowak, L Patino-Studencki, J Robert, A Heuberger, J Thielecke, A low-cost rssi-based localization system for wildlife tracking. IOP Conf. Ser. Mater. Sci. Eng. 120(1), 012004 (2016). https://doi.org/10. 1088/1757-899X/120/1/012004

20. T Nowak, M Hartmann, T Lindner, J Thielecke, in IPIN 2015 Sixth International Conference on Indoor Positioning and Indoor Navigation (IIIN 2015). Optimal network topology for a locating system using rssi-based direction finding, (Banff, Canada, 2015)

21. S Kay, Fundamentals of statistical signal processing. (Prentice-Hall PTR, Englewood Cliffs, 1993)

22. E Nitzan, T Routtenberg, J Tabrikian, A new class of Bayesian cyclic bounds for periodic parameter estimation. IEEE Trans. Signal Process. 64(1), 229-243 (2016). https://doi.org/10.1109/TSP.2015.2478758

23. T Routtenberg, J Tabrikian, in 2016 19th International Conference on Information Fusion (FUSION). Cyclic Cramer-Rao-type bounds for periodic parameter estimation, (2016), pp. 1797-1804
24. T Nowak, M Hartmann, L Patino-Studencki, J Thielecke, in 201613 th Workshop on Positioning, Navigation and Communications (WPNC) Fundamental limits in rssi-based direction-of-arrival estimation, (2016), pp. 1-6. https://doi.org/10.1109/WPNC.2016.7822837

25. CA Balanis, Modern antenna handbook. (Wiley-Interscience, New York, 2008)

26. DG Manolakis, VK Ingle, SM Kogon, Statistical and adaptive signal processing: spectral estimation, signal modeling, adaptive filtering, and array processing vol. 46. (Artech House Norwood, 685 Canton Street, Norwood, 2005)

27. T Nowak, M Hartmann, T Zech, J Thielecke, in IEEE-APS Topical Conference on Antennas and Propagation in Wireless Communications (IEEE-APWC 2016). A path loss and fading model for rssi-based localization in forested areas, (2016). https://doi.org/10.1109/APWC.2016.7738133

28. J Xu, M Ma, CL Law, in Global Telecommunications Conference, 2008. IEEE GLOBECOM 2008. IEEE. AOA cooperative position localization, (2008), pp. 1-5. https://doi.org/10.1109/GLOCOM.2008.ECP.720

29. K Yu, I Sharp, YJ Guo, Ground-based wireless positioning, 1st edn. (Wiley-IEEE Press, 111 River St, Hoboken, 2009)

30. HL Van Trees, Detection, estimation, and modulation theory. (John Wiley \& Sons, Hoboken, 2004)

31. P Misra, P Enge, Global positioning system: signals, measurements, and performance. (Ganga-Jamuna Press, Lincoln, 2006)

32. PCR Drane, Positioning systems: a unified approach, 1st edn, Lecture Notes in Control and Information Sciences 181. (Springer, 233 Spring Street, New York, 1992)

33. SM Sowelam, AH Tewfik, Waveform selection in radar target classification. IEEE Trans. Inf. Theory. 46(3), 1014-1029 (2000). https://doi.org/10.1109/ 18.841178

34. (TE Tuncer, B Friedlander, eds.), Classical and modern direction-of-arrival estimation. (Academic Press, Burlington, 2009)

35. CE Shannon, W Weaver, The mathematical theory of information. (University of Illinois Press, Urbana, 1949)

36. DJC MacKay, Information theory, inference and learning algorithms. (Cambridge University Press, New York, 2002)

37. KV Mardia, PJ Zemroch, Algorithm as 86: the von Mises distribution function. J. R. Stat. Soc. Ser. C: Appl. Stat. 24(2), 268-272 (1975)

38. AV Lazo, P Rathie, On the entropy of continuous probability distributions (corresp.) IEEE Trans. Inf. Theory. 24(1), 120-122 (1978). https://doi.org/10. 1109/TIT.1978.1055832

39. KV Mardia, PE Jupp, Directional Statistics, vol. 494. (John Wiley \& Sons, 111 River St, Hoboken, 2009)

\section{Submit your manuscript to a SpringerOpen ${ }^{\odot}$ journal and benefit from:}

- Convenient online submission

- Rigorous peer review

- Open access: articles freely available online

- High visibility within the field

- Retaining the copyright to your article

Submit your next manuscript at $\gg$ springeropen.com 